\title{
Le dialogue entre médecin et patient facilite le diagnostic de la dépression parfois masquée
}

Le Prix de recherche 2012 du Collège de médecine de premier recours (CMPR) distingue deux études axées sur le dialogue entre le médecin et ses patients. La remise des prix a eu lieu le 21 juin au KKL de Lucerne, dans le cadre de la 14e Journée de formation continue du KHM/CMPR.

Winfried Suske

1 Lombardo $\mathrm{P}$, Vaucher $\mathrm{P}$ Haftgoli N, Burnand B, Favrat $B$, Verdon F, Bischoff T, Herzig L. The help question doesn't help when screening for major depression: external validation of the three question screening tes for primary care patients managed for physical complaints. BMC Medicine. 2011;9:114.

2 Whooley MA, Avins AL Miranda J, Browner WS Case-finding instruments for depression. Two questions are as good as many. J Gen Intern Med. 1997;12:439-445.

3 Arroll B, Goodyear-Smith F, Kerse N, Fishman T, Gunn J. Effect of the addition of a «help» question to two screening questions on specificity for diagnosis of depression in general practice: diagnostic validity study. BMJ. 2005; 331:884

4 Litschgi L. Der Arzt auf dem Sozius - tolerieren statt dirigieren. PrimaryCare. 2012;12:7-10

Dr Winfried Suske c/o Healthworld (Schweiz) AG 6132 Steinhausen Tél. 0417487629

winfried.suske[at]healthworld.ch

\section{Prix principal}

Le prix principal du CMPR, doté de 25000 francs, a été décerné à Patrick Lombardo et à l'équipe de recherche dirigée par le Dr Lilli Herzig de l'Institut Universitaire de Médecine Générale de Lausanne (IUMG), pour leur étude [1] sur les questions de dépistage de dépressions par les médecins de premier recours.

Parmi treize projets de recherche, «nous avons sélectionné cette étude, conçue et réalisée par des médecins généralistes en collaboration avec un centre universitaire, pour son excellence et ses applications pratiques directes pour tous les médecins. Les dépressions sont fréquemment rencontrées dans la pratique du généraliste. Elles sont cependant difficiles à diagnostiquer, car souvent masquées par des symptômes somatiques inexpliqués qui sont généralement l'unique raison de consultation», constate le président du jury Hans Stalder, professeur honoraire de l'Université de Genève.

\section{Le médecin de famille face à la dépression}

Dans le cadre de cette recherche, menée entre novembre 2004 et juillet 2005 en Suisse romande en collaboration avec 24 médecins de premier recours, deux questions simples de dépistage des dépressions (initialement proposées par Whooley et al. [2]) ont été posées aux patients inclus dans l'étude par leur médecin:1. «Est-ce que durant le mois qui a précédé vous êtesvous senti(e) souvent triste, déprimé(e), désespéré(e)?»; 2. «Durant le mois qui a précédé, avez-vous ressenti un manque d'intérêt et de plaisir dans la plupart des activités que d'habitude vous appréciez?»

Le protocole de recherche a été complété avec la «help» question : «Souhaitez-vous de l'aide pour cela?», en tant que troisième question de dépistage proposée à la même période par Arroll et al. [3] .

\section{Neuf cas de dépressions sur dix repérés avec deux questions}

L'analyse des réponses des 724 patients qui ont accepté de répondre aux trois questions au début et à la fin de l'étude montre que les deux premières questions permettent de détecter une dépression majeure dans neuf cas sur dix. Le test est considéré positif lorsque le patient a répondu positivement à l'une des deux questions au moins. Les deux questions de dépistage sont simples, très rapides et faciles à intégrer dans une consultation médicale. L'étude a démontré une prévalence de la dépression majeure chez 9,5\% des patients consultant leur médecin pour des plaintes somatiques.

Par contre, en ajoutant la «help» question, la sensibilité du dépistage est diminuée: quatre patients dépressifs majeurs sur dix déclarent ne pas avoir besoin d'aide. En conséquence, la «help» question ne devrait pas être inclue dans l'outil de dépistage. Elle peut cependant être utile pour le suivi, une fois que la dépression est confirmée, car elle permet de mieux cibler les patients intéressés à la prise en charge de leur dépression.

\section{Prix spécial}

Le jury a accordé un prix spécial au Dr Louis Litschgi, médecin généraliste installé à Bâle, pour son étude originale de suivi de patients qui n'étaient pas d'accord avec la thérapie proposée par le médecin [4]. L'étude analyse la question: «Que se passe-t-il si le patient choisit sciemment une procédure non conforme aux connaissances médicales actuelles?» Pour y répondre, le Dr Litschgi a suivi, avec quelques collègues, 80 cas sur une durée de six années.

Dans cette médecine «participative», les médecins ont laissé le patient prendre la direction et l'ont assisté dans ses choix thérapeutiques, même s'ils paraissent risqués du point de vue médical. Les résultats sont parlants: chez $27 \%$ des patients le taux de guérison était meilleur qu'il ne l'aurait été avec un traitement conventionnel. Seul chez 14\% des patients le taux de guérison était moins satisfaisant. Et les coûts de santé étaient moins chers dans deux tiers des cas. De plus, la satisfaction était grande chez les médecins tout comme chez les patients impliqués dans l'étude.

Vous trouverez des articles détaillés au sujet du CMPR Prix de recherche 2012 dans l'édition actuelle de PrimaryCare ( $\left.N^{\circ} 13 / 2012\right)$, www.primary-care.ch 